\title{
Hadronic light-by-light scattering in the muon $g-2$ : a new short-distance constraint on pion exchange
}

\author{
Andreas Nyffeler \\ Regional Centre for Accelerator-based Particle Physics \\ Harish-Chandra Research Institute \\ Chhatnag Road, Jhusi \\ Allahabad - 211019 , India \\ E-mail: nyffelerahri.res.in
}

\begin{abstract}
We summarize our recent new evaluation of the pion-exchange contribution to hadronic lightby-light scattering in the muon $g-2$. We first derive a new short-distance constraint on the off-shell pion-photon-photon form factor at the external vertex in $a_{\mu}$ which relates the form factor to the quark condensate magnetic susceptibility in QCD. We then evaluate the pion-exchange contribution in the framework of large- $N_{C}$ QCD using an off-shell form factor which fulfills all short-distance constraints and obtain the new estimate $a_{\mu}^{\mathrm{LbyL} ; \pi^{0}}=(72 \pm 12) \times 10^{-11}$. Updating our earlier results for the contributions from the exchanges of the $\eta$ and $\eta^{\prime}$ using simple vectormeson dominance form factors, we get $a_{\mu}^{\text {LbyL;PS }}=(99 \pm 16) \times 10^{-11}$ for the sum of all light pseudoscalars. Combined with available evaluations for the other contributions to hadronic lightby-light scattering this leads to the estimate $a_{\mu}^{\text {LbyL;had }}=(116 \pm 40) \times 10^{-11}$. The corresponding contributions to the anomalous magnetic moment of the electron are also given.
\end{abstract}

PACS: $13.40 . \mathrm{Em}, 12.38 . \mathrm{Lg}, 14.40 . \mathrm{Aq}, 14.60 . \mathrm{Ef}$

6th International Workshop on Chiral Dynamics

July 6-10, 2009

Bern, Switzerland 


\section{Introduction}

The muon $g-2$ has served over many decades as an important test of the Standard Model (SM). It is also sensitive to contributions from New Physics slightly above the electroweak scale. In fact, for several years now a discrepancy of more than three standard deviations has existed between the SM prediction and the experimental value, see the recent reviews Refs. [1, 2, 3] on the muon $g-2$. The main error in the theoretical SM prediction comes from hadronic contributions, i.e. hadronic vacuum polarization and hadronic light-by-light (had. LbyL) scattering. Whereas the hadronic vacuum polarization contribution can be related to the cross section $e^{+} e^{-} \rightarrow$ hadrons, no direct experimental information is available for had. LbyL scattering. One therefore has to rely on hadronic models to describe the strongly interacting, nonperturbative dynamics at the relevant scales from the muon mass up to about $2 \mathrm{GeV}$. This leads to large uncertainties, see Refs. [4, 5, 3] for recent reviews on had. LbyL scattering, largely based on the original works $[6,7,8,9]$.

Essentially, these models describe the interactions of hadrons with photons, usually with the help of some form factors. One can reduce this model dependence and the corresponding uncertainties by relating the form factors at low energies to results from chiral perturbation theory (ChPT) [10] and at high energies (short distances) to the operator product expansion (OPE) [11]. In this way, one connects the form factors to the underlying theory of QCD. In particular, this has been done in Refs. $[6,7,12,8,9]$ for the numerically dominant contribution from the exchange of light pseudoscalars $\pi^{0}, \eta, \eta^{\prime}$.

The pseudoscalar-exchange contributions to had. LbyL scattering are given by the diagrams shown in Fig. 1.
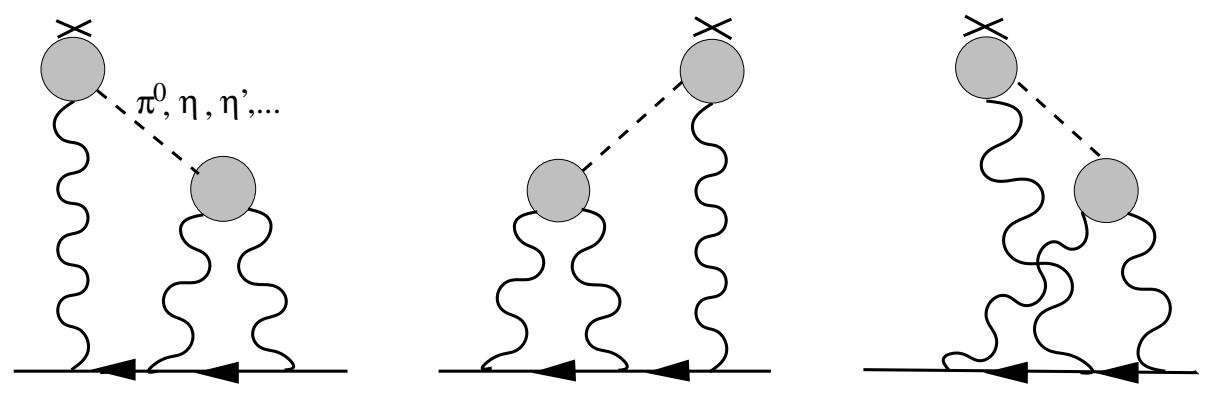

Figure 1: The pseudoscalar-exchange contributions to had. LbyL scattering. The shaded blobs represent the form factor $\mathscr{F}_{\mathrm{PS}^{*} \gamma^{*} \gamma^{*}}$ where PS $=\pi^{0}, \eta, \eta^{\prime}, \pi^{0^{\prime}}, \ldots$

It was pointed out recently in Ref. [2], that one should use fully off-shell form factors for the evaluation of the LbyL scattering contribution. This seems to have been overlooked in the recent literature, in particular, in Refs. [12, 8, 9, 4, 5]. The on-shell form factors as used in Refs. [8, 12] actually violate four-momentum conservation at the external vertex, as observed already in Ref. [9].

The exchange of the lightest state $\pi^{0}$ yields the largest contribution and therefore warrants special attention. In these proceedings, based on the results obtained in Ref. [13], we present a new QCD short-distance constraint on the off-shell pion-photon-photon form factor $\mathscr{F}_{\pi^{0 *}} \gamma^{*} \gamma^{*}$ at the external vertex by relating it to the quark condensate magnetic susceptibility of QCD. We then evaluate this contribution in the framework of large- $N_{C}$ QCD [14], using a form factor which fulfills this new and other relevant short-distance constraints. 


\section{On-shell versus off-shell form factors}

For the pion, the key object which enters the diagrams in Fig. 1 is the off-shell form factor $\mathscr{F}_{\pi^{0}{ }^{*} \gamma^{*}}\left(\left(q_{1}+q_{2}\right)^{2}, q_{1}^{2}, q_{2}^{2}\right)$ which can be defined via the QCD Green's function $\langle V V P\rangle[6,7,13]$

$$
\begin{aligned}
& \int d^{4} x d^{4} y e^{i\left(q_{1} \cdot x+q_{2} \cdot y\right)}\left\langle 0\left|T\left\{j_{\mu}(x) j_{v}(y) P^{3}(0)\right\}\right| 0\right\rangle \\
& =\varepsilon_{\mu v \alpha \beta} q_{1}^{\alpha} q_{2}^{\beta} \frac{i\langle\bar{\psi} \psi\rangle}{F_{\pi}} \frac{i}{\left(q_{1}+q_{2}\right)^{2}-m_{\pi}^{2}} \mathscr{F}_{\pi^{0^{*}} \gamma^{*} \gamma^{*}}\left(\left(q_{1}+q_{2}\right)^{2}, q_{1}^{2}, q_{2}^{2}\right)+\ldots
\end{aligned}
$$

up to small mixing effects with the states $\eta$ and $\eta^{\prime}$ and neglecting exchanges of heavier states like $\pi^{0^{\prime}}, \pi^{0^{\prime \prime}}, \ldots$ Here $j_{\mu}(x)$ is the light quark part of the electromagnetic current and $P^{3}=\bar{\psi} i \gamma_{5} \frac{\lambda^{3}}{2} \psi$.

The corresponding contribution to the muon $g-2$ may be worked out with the result [8]

$$
\begin{aligned}
a_{\mu}^{\mathrm{LbyL} ; \pi^{0}}= & -e^{6} \int \frac{d^{4} q_{1}}{(2 \pi)^{4}} \frac{d^{4} q_{2}}{(2 \pi)^{4}} \frac{1}{q_{1}^{2} q_{2}^{2}\left(q_{1}+q_{2}\right)^{2}\left[\left(p+q_{1}\right)^{2}-m_{\mu}^{2}\right]\left[\left(p-q_{2}\right)^{2}-m_{\mu}^{2}\right]} \\
\times & {\left[\frac{\mathscr{F}_{\pi^{0} \gamma^{*} \gamma^{*}}\left(q_{2}^{2}, q_{1}^{2},\left(q_{1}+q_{2}\right)^{2}\right) \mathscr{F}_{\pi^{0}} \gamma^{*} \gamma}{\left.q_{2}^{2}-m_{\pi}^{2}, q_{2}^{2}, 0\right)} T_{1}\left(q_{1}, q_{2} ; p\right)\right.} \\
& \left.+\frac{\mathscr{F}_{\pi^{0} \gamma^{*} \gamma^{*}}\left(\left(q_{1}+q_{2}\right)^{2}, q_{1}^{2}, q_{2}^{2}\right) \mathscr{F}_{\pi^{0} \gamma^{*} \gamma}\left(\left(q_{1}+q_{2}\right)^{2},\left(q_{1}+q_{2}\right)^{2}, 0\right)}{\left(q_{1}+q_{2}\right)^{2}-m_{\pi}^{2}} T_{2}\left(q_{1}, q_{2} ; p\right)\right],
\end{aligned}
$$

where the external photon has now zero four-momentum. See Ref. [8] for the expressions for $T_{i}$.

Instead of the representation in Eq. (2.2), Refs. [12, 8] considered on-shell form factors which would yield the so called pion-pole contribution, e.g. for the term involving $T_{2}$, one would write [2]

$$
\mathscr{F}_{\pi^{0} \gamma^{*} \gamma^{*}}\left(m_{\pi}^{2}, q_{1}^{2}, q_{2}^{2}\right) \times \mathscr{F}_{\pi^{0} \gamma^{*} \gamma}\left(m_{\pi}^{2},\left(q_{1}+q_{2}\right)^{2}, 0\right) .
$$

Although pole dominance might be expected to give a reasonable approximation, it is not correct as it was used in those references, as stressed in Refs. $[9,2]$. The point is that the form factor sitting at the external photon vertex in the pole approximation $\mathscr{F}_{\pi^{0} \gamma^{*} \gamma}\left(m_{\pi}^{2},\left(q_{1}+q_{2}\right)^{2}, 0\right)$ for $\left(q_{1}+q_{2}\right)^{2} \neq m_{\pi}^{2}$ violates four-momentum conservation, since the momentum of the external (soft) photon vanishes. The latter requires $\mathscr{F}_{\pi^{*} \gamma^{*} \gamma}\left(\left(q_{1}+q_{2}\right)^{2},\left(q_{1}+q_{2}\right)^{2}, 0\right)$. Ref. [9] then proposed to use instead

$$
\mathscr{F}_{\pi^{0} \gamma^{*} \gamma^{*}}\left(m_{\pi}^{2}, q_{1}^{2}, q_{2}^{2}\right) \times \mathscr{F}_{\pi^{0} \gamma \gamma}\left(m_{\pi}^{2}, m_{\pi}^{2}, 0\right) .
$$

Note that putting the pion on-shell at the external vertex automatically leads to a constant form factor, given by the Wess-Zumino-Witten (WZW) term [15]. However, this prescription does not yield the pion-exchange contribution with off-shell form factors, which we calculate with Eq. (2.2).

Strictly speaking, the identification of the pion-exchange contribution is only possible, if the pion is on-shell. If one is off the mass shell of the exchanged particle, it is not possible to separate different contributions to the $g-2$, unless one uses some particular model where elementary pions can propagate. In this sense, only the pion-pole contribution with on-shell form factors can be defined, at least in principle, in a model-independent way. On the other hand, the pion-pole contribution is only a part of the full result, since in general, e.g. using some resonance Lagrangian, the form factors will enter the calculation with off-shell momenta. In this respect, we view our evaluation as being a part of a full calculation of had. LbyL scattering using a resonance Lagrangian whose coefficients are tuned in such a way as to systematically reproduce the relevant QCD shortdistance constraints, along the lines of the resonance chiral theory developed in Ref. [16]. 


\section{A new short-distance constraint on the off-shell pion-photon-photon form factor}

The form factor $\mathscr{F}_{\pi^{0} \gamma^{*} \gamma^{*}}\left(\left(q_{1}+q_{2}\right)^{2}, q_{1}^{2}, q_{2}^{2}\right)$ defined in Eq. (2.1) is determined by nonperturbative physics of QCD and cannot (yet) be calculated from first principles. Therefore, various hadronic models have been used in the literature. At low energies, the form factor is normalized by the decay amplitude, $\mathscr{A}\left(\pi^{0} \rightarrow \gamma \gamma\right) \equiv e^{2} \mathscr{F}_{\pi^{0} \gamma \gamma}\left(m_{\pi}^{2}, 0,0\right)$. To a good approximation, all hadronic models thus have to satisfy the constraint $\mathscr{F}_{\pi^{0} \gamma \gamma}\left(m_{\pi}^{2}, 0,0\right)=-N_{C} /\left(12 \pi^{2} F_{\pi}\right){ }^{1}$

For an on-shell pion, there is also experimental data available for one on-shell and one off-shell photon, from the process $e^{+} e^{-} \rightarrow e^{+} e^{-} \pi^{0}$. Several experiments [19] thereby fairly well confirm the Brodsky-Lepage [20] behavior for large Euclidean momentum $\lim _{Q^{2} \rightarrow \infty} \mathscr{F}_{\pi^{0} \gamma^{*} \gamma}\left(m_{\pi}^{2},-Q^{2}, 0\right) \sim$ $-2 F_{\pi} / Q^{2}$ and any model should reproduce this behavior, maybe with a different prefactor. ${ }^{2}$

Apart from these experimental constraints, any consistent hadronic model for the off-shell form factor $\mathscr{F}_{\pi^{0} \gamma^{*} \gamma^{*}}\left(\left(q_{1}+q_{2}\right)^{2}, q_{1}^{2}, q_{2}^{2}\right)$ should match at large momentum with short-distance constraints from QCD that can be calculated using the OPE. In Ref. [22] the short-distance properties for the three-point function $\langle V V P\rangle$ in Eq. (2.1) in the chiral limit and assuming octet symmetry have been worked out in detail. Two limits are of interest. In the first case, the two momenta become simultaneously large, which describes the situation where the space-time arguments of all three operators tend towards the same point at the same rate. The second situation corresponds to the case where the relative distance between only two of the three operators in $\langle V V P\rangle$ becomes small. When the space-time arguments of the two vector currents in $\langle V V P\rangle$ approach each other, the leading term in the OPE leads to the Green's function $\langle A P\rangle$. The explicit results for both these cases can be found in Refs. [22, 13].

The new short-distance constraint on the off-shell form factor at the external vertex in had. LbyL scattering arises when the space-time argument of one of the vector currents in $\langle V V P\rangle$ approaches the argument of the pseudoscalar density. This leads to the two-point function $\langle V T\rangle$ of the vector current and the antisymmetric tensor density

$$
\delta^{a b}\left(\Pi_{\mathrm{VT}}\right)_{\mu \rho \sigma}(p)=\int d^{4} x e^{i p \cdot x}\left\langle 0\left|T\left\{V_{\mu}^{a}(x)\left(\bar{\psi} \sigma_{\rho \sigma} \frac{\lambda^{b}}{2} \psi\right)(0)\right\}\right| 0\right\rangle, \quad \sigma_{\rho \sigma}=\frac{i}{2}\left[\gamma_{\rho}, \gamma_{\sigma}\right]
$$

Conservation of the vector current and invariance under parity then give $\left(\Pi_{\mathrm{VT}}\right)_{\mu \rho \sigma}(p)=\left(p_{\rho} \eta_{\mu \sigma}-\right.$ $\left.p_{\sigma} \eta_{\mu \rho}\right) \Pi_{\mathrm{VT}}\left(p^{2}\right)$. In this way one obtains the relation (up to corrections of order $\alpha_{s}$ ) $[22,13]$

$$
\lim _{\lambda \rightarrow \infty} \mathscr{F}_{\pi^{0^{*}} \gamma^{*} \gamma^{*}}\left(\left(\lambda q_{1}+q_{2}\right)^{2},\left(\lambda q_{1}\right)^{2}, q_{2}^{2}\right)=-\frac{2}{3} \frac{F_{0}}{\langle\bar{\psi} \psi\rangle_{0}} \Pi_{\mathrm{VT}}\left(q_{2}^{2}\right)+\mathscr{O}\left(\frac{1}{\lambda}\right)
$$

In particular, at the external vertex in LbyL scattering in Eq. (2.2), the limit $q_{2} \rightarrow 0$ is relevant.

As pointed out in Ref. [23], the value of $\Pi_{\mathrm{VT}}\left(p^{2}\right)$ at zero momentum is related to the quark condensate magnetic susceptibility $\chi$ in QCD in the presence of a constant external electromagnetic field, introduced in Ref. [24]: $\left\langle 0\left|\bar{q} \sigma_{\mu \nu} q\right| 0\right\rangle_{F}=e e_{q} \chi\langle\bar{\psi} \psi\rangle_{0} F_{\mu \nu}$, with $e_{u}=2 / 3$ and $e_{d}=-1 / 3$.

\footnotetext{
${ }^{1}$ We note that in our work [13] and in Refs. $[6,7,8,9]$ simply $F_{\pi}=92.4 \mathrm{MeV}$ is used, without any error attached. Maybe this could be an additional source of uncertainty in $a_{\mu}^{\mathrm{LbyL} ; \pi^{0}}$, in particular in view of the new value $\Gamma\left(\pi^{0} \rightarrow \gamma \gamma\right)=$ $(7.82 \pm 0.23) \mathrm{eV}$ presented in Ref. [17]; see also the discussion in Ref. [18] and references therein.

${ }^{2}$ Note, however, that a recent measurement of the form factor by the BABAR collaboration [21] at momentum transfers $Q^{2}$ between $4 \mathrm{GeV}^{2}$ and $40 \mathrm{GeV}^{2}$ does not show such a falloff. We will come back to this issue in Section 4.
} 
With our definition of $\Pi_{\mathrm{VT}}$ in Eq. (3.1) one obtains the relation $\Pi_{\mathrm{VT}}(0)=-\left(\langle\bar{\psi} \psi\rangle_{0} / 2\right) \chi$ (see also Ref. [25]) and the new short-distance constraint at the external vertex can be written as [13]

$$
\lim _{\lambda \rightarrow \infty} \mathscr{F}_{\pi^{0} \gamma^{*} \gamma}\left(\left(\lambda q_{1}\right)^{2},\left(\lambda q_{1}\right)^{2}, 0\right)=\frac{F_{0}}{3} \chi+\mathscr{O}\left(\frac{1}{\lambda}\right) .
$$

Note that there is no falloff in this limit, unless $\chi$ vanishes.

Unfortunately there is no agreement in the literature what the actual value of $\chi$ should be. In comparing different results one has to keep in mind that $\chi$ actually depends on the renormalization scale $\mu$. In Ref. [24] the estimate $\chi(\mu=0.5 \mathrm{GeV})=-\left(8.16_{-1.91}^{+2.95}\right) \mathrm{GeV}^{-2}$ was given in a QCD sum rule evaluation of nucleon magnetic moments. A similar value $\chi=-N_{C} /\left(4 \pi^{2} F_{\pi}^{2}\right)=-8.9 \mathrm{GeV}^{-2}$ was obtained in Ref. [26], probably again for a low scale $\mu \sim 0.5 \mathrm{GeV}$ as argued in Ref. [26].

On the other hand, saturating the leading short-distance behavior of the two-point function $\Pi_{\mathrm{VT}}$ [27] with one multiplet of lowest-lying vector mesons (LMD) [28, 23, 22] leads to the estimate $\chi^{\mathrm{LMD}}=-2 / M_{V}^{2}=-3.3 \mathrm{GeV}^{-2}$ [28]. Again, it is not obvious at which scale this relation holds, it might be at $\mu=M_{V}$. This LMD estimate was soon afterwards improved by taking into account higher resonance states $\left(\rho^{\prime}, \rho^{\prime \prime}\right)$ in the framework of QCD sum rules, with the results $\chi(0.5 \mathrm{GeV})=$ $-(5.7 \pm 0.6) \mathrm{GeV}^{-2}[23]$ and $\chi(1 \mathrm{GeV})=-(4.4 \pm 0.4) \mathrm{GeV}^{-2}$ [29]. A more recent analysis [30] yields, however, a smaller absolute value $\chi(1 \mathrm{GeV})=-(3.15 \pm 0.30) \mathrm{GeV}^{-2}$, close to the original LMD estimate. ${ }^{3}$ For a quantitative comparison of all these estimates for $\chi$ we would have to run them to a common scale, for instance, $1 \mathrm{GeV}$ or $2 \mathrm{GeV}$, which can obviously not be done within perturbation theory starting from such low scales as $\mu=0.5 \mathrm{GeV}$.

\section{New evaluation of the pseudoscalar-exchange contribution in large- $N_{C} \mathbf{Q C D}$}

In the spirit of the minimal hadronic Ansatz [33] for Green's functions in large- $N_{C} \mathrm{QCD}$, an off-shell form factor $\mathscr{F}_{\pi^{*} \gamma^{*} \gamma^{*}}\left(\left(q_{1}+q_{2}\right)^{2}, q_{1}^{2}, q_{2}^{2}\right)$ has been constructed in Ref. [22]. It contains the two lightest multiplets of vector resonances, the $\rho$ and the $\rho^{\prime}(\mathrm{LMD}+\mathrm{V})$, and fulfills all the OPE constraints discussed earlier:

$$
\begin{aligned}
\underset{\pi^{0^{*}} \gamma^{*} \gamma^{*}}{\mathrm{LM}+\mathrm{V}}\left(q_{3}^{2}, q_{1}^{2}, q_{2}^{2}\right)= & \frac{F_{\pi}}{3} \frac{q_{1}^{2} q_{2}^{2}\left(q_{1}^{2}+q_{2}^{2}+q_{3}^{2}\right)+P_{H}^{V}\left(q_{1}^{2}, q_{2}^{2}, q_{3}^{2}\right)}{\left(q_{1}^{2}-M_{V_{1}}^{2}\right)\left(q_{1}^{2}-M_{V_{2}}^{2}\right)\left(q_{2}^{2}-M_{V_{1}}^{2}\right)\left(q_{2}^{2}-M_{V_{2}}^{2}\right)}, \\
P_{H}^{V}\left(q_{1}^{2}, q_{2}^{2}, q_{3}^{2}\right)= & h_{1}\left(q_{1}^{2}+q_{2}^{2}\right)^{2}+h_{2} q_{1}^{2} q_{2}^{2}+h_{3}\left(q_{1}^{2}+q_{2}^{2}\right) q_{3}^{2}+h_{4} q_{3}^{4} \\
& +h_{5}\left(q_{1}^{2}+q_{2}^{2}\right)+h_{6} q_{3}^{2}+h_{7}, \quad q_{3}^{2}=\left(q_{1}+q_{2}\right)^{2} .
\end{aligned}
$$

Below we reevaluate the pion-exchange contribution using off-shell LMD+V form factors at both vertices. The constants $h_{i}$ in the Ansatz for $\mathscr{F}_{\pi^{0} \gamma^{*} \gamma^{*}}^{\mathrm{LMD}+\mathrm{V}}$ in Eq. (4.1) are determined as follows. The normalization with the pion decay amplitude $\pi^{0} \rightarrow \gamma \gamma$ yields $h_{7}=-N_{C} M_{V_{1}}^{4} M_{V_{2}}^{4} /\left(4 \pi^{2} F_{\pi}^{2}\right)-$ $h_{6} m_{\pi}^{2}-h_{4} m_{\pi}^{4}=-14.83 \mathrm{GeV}^{6}-h_{6} m_{\pi}^{2}-h_{4} m_{\pi}^{4}$, where we used $M_{V_{1}}=M_{\rho}=775.49 \mathrm{MeV}$ and $M_{V_{2}}=$ $M_{\rho^{\prime}}=1.465 \mathrm{GeV}$. The Brodsky-Lepage behavior can be reproduced by choosing $h_{1}=0 \mathrm{GeV}^{2}$.

\footnotetext{
${ }^{3}$ After the publication of our paper Ref. [13], two new estimates for $\chi$ appeared, both based on the analysis of the zero-modes of the Dirac operator. Ref. [31] presents an analytical approach which yields $\chi(1 \mathrm{GeV})=-3.52 \mathrm{GeV}^{-2}$ with an estimated error of $30-50 \%$. A quenched lattice calculation [32] for $N_{C}=2$ gives a very small absolute value $\chi=-1.547(6) \mathrm{GeV}^{-2}$. No scale dependence is given, the lattice spacing corresponds to $2 \mathrm{GeV}$.
} 
In Ref. [22] a fit to the CLEO data [19] for the on-shell form factor $\mathscr{F}_{\pi^{0} \gamma^{*} \gamma}^{\mathrm{LMD}+\mathrm{V}}\left(m_{\pi}^{2},-Q^{2}, 0\right)$ was performed, with the result $h_{5}=(6.93 \pm 0.26) \mathrm{GeV}^{4}-h_{3} m_{\pi}^{2}$. The constant $h_{2}$ can be obtained from higher-twist corrections in the OPE with the result $h_{2}=-10.63 \mathrm{GeV}^{2}$ [9].

Within the LMD+V framework, the vector-tensor two-point function reads [22, 13]

$$
\Pi_{\mathrm{VT}}^{\mathrm{LMD}+\mathrm{V}}\left(p^{2}\right)=-\langle\bar{\psi} \psi\rangle_{0} \frac{p^{2}+c_{\mathrm{VT}}}{\left(p^{2}-M_{V_{1}}^{2}\right)\left(p^{2}-M_{V_{2}}^{2}\right)}, \quad c_{\mathrm{VT}}=\frac{M_{V_{1}}^{2} M_{V_{2}}^{2} \chi}{2} .
$$

As shown in Ref. [22], the OPE constraint from Eq. (3.2) for $\underset{\pi^{0 *} \gamma^{*} \gamma^{*}}{\mathrm{LMD}+\mathrm{V}}$ leads to the relation

$$
h_{1}+h_{3}+h_{4}=2 c_{\mathrm{VT}} .
$$

The LMD estimate $\chi^{\mathrm{LMD}}=-2 / M_{V}^{2}=-3.3 \mathrm{GeV}^{-2}$ is close to $\chi(\mu=1 \mathrm{GeV})=-(3.15 \pm$ 0.30) $\mathrm{GeV}^{-2}$ obtained in Ref. [30] using QCD sum rules with several vector resonances $\rho, \rho^{\prime}$, and $\rho^{\prime \prime}$. Assuming that the $\mathrm{LMD} / \mathrm{LMD}+\mathrm{V}$ framework is self-consistent, we will therefore take $\chi=(-3.3 \pm 1.1) \mathrm{GeV}^{-2}$ in our numerical evaluation, with a typical large- $N_{C}$ uncertainty of about $30 \%$. We will vary $h_{3}$ in the range $\pm 10 \mathrm{GeV}^{2}$ and determine $h_{4}$ from Eq. (4.4) and vice versa.

The coefficient $h_{6}$ in the LMD+V Ansatz is undetermined as well. It enters at order $p^{6}$ in the low-energy expansion of $\langle V V P\rangle$ in one combination of low-energy constants from the chiral Lagrangian of odd intrinsic parity, $A_{V,(p+q)^{2}}^{\mathrm{LMD}+\mathrm{V}}=-F_{\pi}^{2} h_{6} /\left(8 M_{V_{1}}^{4} M_{V_{2}}^{4}\right)$ [22]. The LMD ansatz with only one multiplet of vector resonances yields $A_{V,(p+q)^{2}}^{\mathrm{LMD}}=-F_{\pi}^{2} /\left(8 M_{V}^{4}\right)=-0.26\left(10^{-4} / F_{\pi}^{2}\right)$ [22]. If the $\mathrm{LMD} / \mathrm{LMD}+\mathrm{V}$ framework is self-consistent, the change in these estimates, while going from LMD to $\mathrm{LMD}+\mathrm{V}$, should not be too big. Since the size of this low-energy constant seems to be small compared to another combination of low-energy constants which enters at order $p^{6}$, we allow for a $100 \%$ uncertainty of $A_{V,(p+q)^{2}}^{\mathrm{LMD}}$ and get the range $h_{6}=(5 \pm 5) \mathrm{GeV}^{4}$, see Ref. [13] for details.

The results for $a_{\mu}^{\mathrm{LbyL} ; \pi^{0}}$ for some selected values of $h_{3}, h_{4}$ and $h_{6}$, varied in the ranges discussed above, for $\chi=-3.3 \mathrm{GeV}^{-2}, h_{1}=0 \mathrm{GeV}^{2}, h_{2}=-10.63 \mathrm{GeV}^{2}$ and $h_{5}=6.93 \mathrm{GeV}^{4}-h_{3} m_{\pi}^{2}$ are collected in Table 1, see Refs. [13, 3] for details on the numerics.

\begin{tabular}{|l|c|c|c|}
\hline & $h_{6}=0 \mathrm{GeV}^{4}$ & $h_{6}=5 \mathrm{GeV}^{4}$ & $h_{6}=10 \mathrm{GeV}^{4}$ \\
\hline$h_{3}=-10 \mathrm{GeV}^{2}$ & 68.4 & 74.1 & 80.2 \\
$h_{3}=0 \mathrm{GeV}^{2}$ & 66.4 & 71.9 & 77.8 \\
$h_{3}=10 \mathrm{GeV}^{2}$ & 64.4 & 69.7 & 75.4 \\
\hline$h_{4}=-10 \mathrm{GeV}^{2}$ & 65.3 & 70.7 & 76.4 \\
$h_{4}=0 \mathrm{GeV}^{2}$ & 67.3 & 72.8 & 78.8 \\
$h_{4}=10 \mathrm{GeV}^{2}$ & 69.2 & 75.0 & 81.2 \\
\hline
\end{tabular}

Table 1: Results for $a_{\mu}^{\mathrm{LbyL} ; \pi^{0}} \times 10^{11}$ obtained with the off-shell LMD+V form factor for $\chi=-3.3 \mathrm{GeV}^{-2}$ and the given values for $h_{3}, h_{4}$ and $h_{6}$. When varying $h_{3}$ (upper half of the table), the parameter $h_{4}$ is fixed by the constraint in Eq. (4.4). In the lower half the procedure is reversed.

Varying $\chi$ by $\pm 1.1 \mathrm{GeV}^{-2}$ changes the result for $a_{\mu}^{\mathrm{LbyL} ; \pi^{0}}$ by $\pm 2.1 \times 10^{-11}$ at most. The uncertainty in $h_{6}$ affects the result by up to $\pm 6.4 \times 10^{-11}$. The variation of $a_{\mu}^{\mathrm{LbyL} ; \pi^{0}}$ with $h_{3}$ [with $h_{4}$ determined from the constraint in Eq. (4.4) or vice versa] is much smaller, at most $\pm 2.5 \times 10^{-11}$. In the absence of more information on the values of the constants $h_{3}, h_{4}$ and $h_{6}$, we take the average 
of the results obtained with $h_{6}=5 \mathrm{GeV}^{4}$ for $h_{3}=0 \mathrm{GeV}^{2}$ and for $h_{4}=0 \mathrm{GeV}^{2}$ as our central value: $a_{\mu}^{\mathrm{LbyL} ; \pi^{0}}=72.3 \times 10^{-11}$. Adding all uncertainties from the variations of $\chi, h_{3}$ (or $\left.h_{4}\right), h_{5}$ and $h_{6}$ linearly to cover the full range of values obtained with our scan of parameters, we get $[13,3]$

$$
a_{\mu}^{\mathrm{LbyL} ; \pi^{0}}=(72 \pm 12) \times 10^{-11} .
$$

This value replaces the result obtained in Ref. [8] with on-shell LMD+V form factors at both vertices. We think the $16 \%$ error should fairly well describe the inherent model uncertainty using the off-shell $\mathrm{LMD}+\mathrm{V}$ form factor. In order to facilitate updates of our result in case some of the parameters $h_{i}$ in the LMD+V Ansatz in Eq. (4.1) will be known more precisely, we have given in the Appendix of Ref. [13] a parametrization of $a_{\mu}^{\mathrm{LbyL} ; \pi^{0}}$ for arbitrary coefficients $h_{i}{ }^{4}$

As far as the contribution to $a_{\mu}$ from the exchanges of the other light pseudoscalars $\eta$ and $\eta^{\prime}$ is concerned, it is not so straightforward to apply the above analysis within the LMD+V framework to these resonances. In particular, the short-distance analysis in Ref. [22] was performed in the chiral limit and assumed octet symmetry. We therefore resort to a simplified approach which was also adopted in other works $[6,7,8,9]$ and take a simple VMD form factor normalized to the experimental decay width $\Gamma(\mathrm{PS} \rightarrow \gamma \gamma)$. In this way we obtain the results $a_{\mu}^{\mathrm{LbyL} ; \eta}=14.5 \times 10^{-11}$ and $a_{\mu}^{\text {LbyL } ; \eta^{\prime}}=12.5 \times 10^{-11}$, which update the values given in Ref. [8]. Adding up the contributions from all the light pseudoscalar exchanges, we obtain the estimate [13, 3]

$$
a_{\mu}^{\mathrm{LbyL} ; \mathrm{PS}}=(99 \pm 16) \times 10^{-11},
$$

where we have assumed a $16 \%$ error, as inferred above for the pion-exchange contribution. ${ }^{5}$

\section{Discussion and conclusions}

We would like to stress that although our result for the pion-exchange contribution is not too far from the value $a_{\mu}^{\mathrm{LbyL} ; \pi^{0}-\text { pole }}=(76.5 \pm 6.7) \times 10^{-11}$ given in Ref. [9], this is pure coincidence. We have used off-shell $\mathrm{LMD}+\mathrm{V}$ form factors at both vertices, whereas the authors of Ref. [9] evaluated the pion-pole contribution using the on-shell LMD+V form factor $\mathscr{F}_{\pi^{0} \gamma^{*} \gamma^{*}}\left(q_{1}^{2}, q_{2}^{2}\right) \equiv \mathscr{F}_{\pi^{0} \gamma^{*} \gamma^{*}}\left(m_{\pi}^{2}, q_{1}^{2}, q_{2}^{2}\right)$ at the internal vertex and a constant WZW form factor at the external vertex, see for instance Eq. (18) in Ref. [9]. Since only the pion-pole contribution is considered in Ref. [9], their short-distance constraint cannot be applied to our approach either. However, our ansatz for the pion-exchange contribution agrees qualitatively with the short-distance behavior of the quark-loop derived in Ref. [9], see the discussion in Refs. [13, 3].

\footnotetext{
${ }^{4} \mathrm{~A}$ fit of the on-shell LMD+V form factor to the recent BABAR data [21] yields $h_{1}=(-0.17 \pm 0.02) \mathrm{GeV}^{2}$ and $h_{5}=(6.51 \pm 0.20) \mathrm{GeV}^{4}-h_{3} m_{\pi}^{2}$ with $\chi^{2} / \mathrm{dof}=15.0 / 15=1.0$. In this way we would get the new average value $a_{\mu}^{\mathrm{LbyL} ; \pi^{0}}=71.8 \times 10^{-11}$, i.e. the result is essentially unchanged from Eq. (4.5).

${ }^{5}$ Applying the same procedure to the electron, we get $a_{e}^{\mathrm{LbyL} ; \pi^{0}}=(2.98 \pm 0.34) \times 10^{-14}$ [13]. This number supersedes the value given in Ref. [8]. Note that the naive rescaling $a_{e}^{\mathrm{LbyL} ; \pi^{0}}($ rescaled $)=\left(m_{e} / m_{\mu}\right)^{2} a_{\mu}^{\mathrm{LbyL} ; \pi^{0}}=1.7 \times 10^{-14}$ yields a value which is almost a factor of 2 too small. Our estimates for the other pseudoscalars contributions using VMD form factors at both vertices are $a_{e}^{\mathrm{LbyL} ; \eta}=0.49 \times 10^{-14}$ and $a_{e}^{\mathrm{LbyL} ; \eta^{\prime}}=0.39 \times 10^{-14}$. Therefore we get $a_{e}^{\mathrm{LbyL} ; \mathrm{PS}}=(3.9 \pm 0.5) \times 10^{-14}$, where the relative error of about $12 \%$ is again taken over from the pion-exchange contribution. Assuming that the pseudoscalar contribution yields the bulk of the result of the total had. LbyL scattering correction, we obtain $a_{e}^{\text {LbyL;had }}=(3.9 \pm 1.3) \times 10^{-14}$, with a conservative error of about 30\%, see Ref. [3]. This value was later confirmed in the published version of Ref. [5] where a leading logs estimate yielded $a_{e}^{\text {LbyL;had }}=(3.5 \pm 1.0) \times 10^{-14}$.
} 
Our results for the pion and the sum of all pseudoscalars are about $20 \%$ larger than the values in Refs. [6, 7] which used other hadronic models. An evaluation of the pion-exchange contribution using an off-shell form factor based on a nonlocal chiral quark model yielded $a_{\mu}^{\mathrm{LbyL} ; \pi^{0}}=(65 \pm 2) \times$ $10^{-11}$ [34]. In that model, off-shell effects of the pion always lead to a strong damping in the form factor and the result is therefore smaller than the pion-pole contribution obtained in Ref. [9]. In our model, there are some corners of the parameter space where the result is larger than the pionpole contribution, for instance, we get a maximal value of $a_{\mu}^{\mathrm{LbyL} ; \pi^{0}}=83.3 \times 10^{-11}$ in the scanned region. Very recently, a value of $a_{\mu}^{\mathrm{LbyL} ; \mathrm{PS}}=107 \times 10^{-11}$ with an estimated error of at most $30 \%$ was obtained in Ref. [35] within an AdS/QCD approach.

Combining our result for the pseudoscalars with the evaluation of the axial-vector contribution in Ref. [9] and the results from Ref. [6] for the other contributions, we obtain the estimate [13, 3]

$$
a_{\mu}^{\text {LbyL;had }}=(116 \pm 40) \times 10^{-11}
$$

for the total had. LbyL scattering contribution to the anomalous magnetic moment of the muon. To be conservative, we have added all the errors linearly, as has become customary in recent years. In the very recent review [5] the central values of some of the individual contributions to had. LbyL scattering are adjusted and some errors are enlarged to cover the results obtained by various groups which used different models. The errors are finally added in quadrature to yield the estimate $a_{\mu}^{\mathrm{LbyL} ; \mathrm{had}}=(105 \pm 26) \times 10^{-11}$. Note that the dressed light quark loops are not included as a separate contribution in Ref. [5]. They are assumed to be already covered by using the shortdistance constraint from Ref. [9] on the pseudoscalar-pole contribution. Certainly, more work on the had. LbyL scattering contribution is needed to fully control all the uncertainties.

\section{Acknowledgments}

I would like to thank the organizers of Chiral Dynamics 2009 for their financial support and for providing such a stimulating atmosphere. I am grateful to F. Jegerlehner for pointing out that fully off-shell form factors should be used to evaluate the pion-exchange contribution, for helpful discussions and for numerous correspondences. Furthermore, I would like to thank G. Colangelo, J. Gasser, M. Knecht, H. Leutwyler, P. Minkowski, B. Moussallam, M. Perrottet, A. Pich, J. Portoles, J. Prades, E. de Rafael and A. Vainshtein for illuminating discussions. This work was supported by the Department of Atomic Energy, Government of India, under a 5-Years Plan Project.

\section{References}

[1] J. P. Miller, E. de Rafael, and B. L. Roberts, Rep. Prog. Phys. 70, 795 (2007).

[2] F. Jegerlehner, Acta Phys. Pol. B 38, 3021 (2007); F. Jegerlehner, The Anomalous Magnetic Moment of the Muon, Springer Tracts Mod. Phys. Vol. 226 (Springer, Berlin, 2008).

[3] F. Jegerlehner and A. Nyffeler, Phys. Rept. 477, 1 (2009).

[4] J. Bijnens and J. Prades, Mod. Phys. Lett. A 22, 767 (2007).

[5] J. Prades, E. de Rafael, and A. Vainshtein in Lepton Dipole Moments, B.L. Roberts and W.J. Marciano, (eds) (World Scientific, Singapore, 2009), 309-324, arXiv:0901.0306 [hep-ph]; J. Prades, arXiv:0909.0953 [hep-ph], these proceedings. 
[6] J. Bijnens, E. Pallante, and J. Prades, Phys. Rev. Lett. 75, 1447 (1995); 75, 3781(E) (1995); Nucl. Phys. B474, 379 (1996); B626, 410 (2002).

[7] M. Hayakawa, T. Kinoshita, and A. I. Sanda, Phys. Rev. Lett. 75, 790 (1995); Phys. Rev. D 54, 3137 (1996); M. Hayakawa and T. Kinoshita, Phys. Rev. D 57, 465 (1998); 66, 019902(E) (2002).

[8] M. Knecht and A. Nyffeler, Phys. Rev. D 65, 073034 (2002); M. Knecht et al., Phys. Rev. Lett. 88, 071802 (2002).

[9] K. Melnikov and A. Vainshtein, Phys. Rev. D 70, 113006 (2004).

[10] S. Weinberg, Physica (Amsterdam) 96A, 327 (1979); J. Gasser and H. Leutwyler, Ann. Phys. (N.Y.) 158, 142 (1984); J. Gasser and H. Leutwyler, Nucl. Phys. B250, 465 (1985).

[11] K. G. Wilson, Phys. Rev. 179, 1499 (1969); M. A. Shifman, A. I. Vainshtein, and V. I. Zakharov, Nucl. Phys. B147, 385 (1979); B147, 448 (1979).

[12] J. Bijnens and F. Persson, hep-ph/0106130.

[13] A. Nyffeler, Phys. Rev. D 79, 073012 (2009).

[14] G. 't Hooft, Nucl. Phys. B72, 461 (1974); B75, 461 (1974); E. Witten, Nucl. Phys. B160, 57 (1979).

[15] J. Wess and B. Zumino, Phys. Lett. 37B, 95 (1971); E. Witten, Nucl. Phys. B223, 422 (1983).

[16] G. Ecker et al., Nucl. Phys. B321, 311 (1989); G. Ecker et al., Phys. Lett. B 223, 425 (1989).

[17] A.M. Bernstein, talk at this conference.

[18] K. Kampf and B. Moussallam, arXiv:0901.4688 [hep-ph]; B. Moussallam, talk at this conference.

[19] H. J. Behrend et al. [The CELLO Collaboration], Z. Phys. C 49, 401 (1991); J. Gronberg et al. [The CLEO Collaboration], Phys. Rev. D 57, 33 (1998).

[20] G. P. Lepage and S. J. Brodsky, Phys. Rev. D 22, 2157 (1980); S. J. Brodsky and G. P. Lepage, Phys. Rev. D 24, 1808 (1981).

[21] B. Aubert et al. [The BABAR Collaboration], arXiv:0905.4778 [hep-ex].

[22] M. Knecht and A. Nyffeler, Eur. Phys. J. C 21, 659 (2001).

[23] V. M. Belyaev and Y. I. Kogan, Yad. Fiz. 40, 1035 (1984).

[24] B. L. Ioffe and A. V. Smilga, Nucl. Phys. B232, 109 (1984).

[25] V. Mateu and J. Portoles, Eur. Phys. J. C 52, 325 (2007).

[26] A. Vainshtein, Phys. Lett. B 569, 187 (2003).

[27] N. S. Craigie and J. Stern, Phys. Rev. D 26, 2430 (1982).

[28] I. I. Balitsky and A. V. Yung, Phys. Lett. 129B, 328 (1983).

[29] I. I. Balitsky, A. V. Kolesnichenko, and A. V. Yung, Yad. Fiz. 41, 282 (1985).

[30] P. Ball, V. M. Braun, and N. Kivel, Nucl. Phys. B649, 263 (2003).

[31] B. L. Ioffe, Phys. Lett. B 678, 512 (2009).

[32] P. V. Buividovich et al., arXiv:0906.0488 [hep-lat].

[33] B. Moussallam and J. Stern, hep-ph/9404353; B. Moussallam, Phys. Rev. D 51, 4939 (1995); B. Moussallam, Nucl. Phys. B504, 381 (1997); S. Peris, M. Perrottet, and E. de Rafael, J. High Energy Phys. 05 (1998) 011; M. Knecht et al., Phys. Rev. Lett. 83, 5230 (1999).

[34] A. E. Dorokhov and W. Broniowski, Phys. Rev. D 78, 073011 (2008).

[35] D. K. Hong and D. Kim, arXiv:0904.4042 [hep-ph]. 